\title{
DESWELLING OF POLY (N-ISOPROPYLACRYLAMIDE) DERIVED HYDROGELS AND THEIR NANOCOMPOSITES WITH IRON OXIDE NANOPARTICLES AS REVEALED BY X-RAY PHOTON CORRELATION SPECTROSCOPY
}

Rebeca Hernández ${ }^{\mathrm{a}, *}$, Miryam Criado ${ }^{\mathrm{a}}$, Aurora Nogales ${ }^{\mathrm{b}}$, Michael Sprung ${ }^{\mathrm{c}}$, Carmen Mijangos $^{\mathrm{a}}$, Tiberio A. Ezquerra ${ }^{\mathrm{a}}$

a Instituto de Ciencia y Tecnología de Polímeros, ICTP-CSIC, Juan de la Cierva, 3, 28006 Madrid, Spain

${ }^{\mathrm{b}}$ Instituto de Estructura de la Materia, IEM-CSIC, Serrano 121, 28006 Madrid, Spain

${ }^{c}$ Petra III at DESY, Notkestr. 85, 22607 Hamburg, Germany

*To whom correspondence should be addressed: rhernandez@ictp.csic.es 


\section{ABSTRACT}

Here we present results on the deswelling behavior of semiinterpenetrating polymer hydrogels of sodium alginate and poly (N-isopropylacrylamide) (PNIPAAm) containing iron oxide nanoparticles by means of X-ray photocorrelation spectroscopy (XPCS). The experiments were carried out as a function of temperature starting from temperatures below the low critical solution temperature (LCST) of the materials to temperatures above their LCST. The hydrogels present a hyper-diffusive dynamics of similar nature as that found for the freeze-dried aerogels which has been attributed to the characteristic porous structure. The dynamics of the swollen samples is faster than that of the freezedried ones suggesting that water has an effect in accelerating the dynamics of the hydrogels. XPCS has been used to characterize the deswelling process as a function of temperature. Deswelling times for the different samples correlate well with the corresponding relaxation times of the relaxation processes measured by XPCS. 


\section{INTRODUCTION}

Thermosensitive hydrogels based on poly (N-isopropylacrylamide) (PNIPAAm) or related copolymers are widely known for their reversible hydration-dehydration change in response to small temperature changes around its lower critical solution temperature (LCST) which is found $\sim 32{ }^{\circ} \mathrm{C}$. Above LCST, the hydrophilic PNiPAAm changes its philicity and becomes hydrophobic so that the polymer gel collapse ${ }^{1}$. Due to the release of hydrophobic hydration, the LCST transition leads to dramatic changes in physical properties, such as mechanical, optical, and thermal properties ${ }^{2}$. Hence, the study of the LCST behaviour of PNiPAAm gels and related copolymers is a very important problem extensively studied, both from a fundamental point of view and because of its relevance for the development of a large variety of applications mainly in biomedicine ${ }^{3,4}$.

X-ray photocorrelation spectroscopy has been employed to determine the dynamics of hydrated PNiPAAm gels at temperatures above the LCST of the material. The results obtained show hyper-diffusive dynamics of PNiPAAm gels and support the notion that the ultraslow deswelling rate observed at the volume phase transition in PNIPAAm hydrogels is the result of jamming ${ }^{5}$. This phenomenon is intrinsic to the polymer structure and is not attributable to its hydrophobic character in the high temperature regime. In fact, an increase in the crosslinking density leads to a higher number of structural inhomogeneities in the gel which, in turn, facilitates rapid deswelling ${ }^{6}$. Typically jammed systems consist of many constituent particles that interact by shortrange repulsive forces. Jamming takes place when the geometrical constraints among constituent elements arrest the dynamics ${ }^{7}$.

The study of the deswelling kinetics of PNiPAAm gels at temperatures above LCST has important implications for the development of applications for these materials. In fact, 
conventional PNiPAAm hydrogels present a slow response rate at temperatures above LCST which restricts wider applications such as on-off valves, artificial muscles or drug delivery applications ${ }^{8}$. In order to increase the response rate of PNiPAAm hydrogels, several successful strategies have been worked out. One of them is to generate pores in the bulk material that allow water molecules to be quickly squeezed out of the gel. This can be done through a process of freeze-drying ${ }^{9}$, incorporation of PNiPAAm nanoparticles $^{10}$ or inorganic microparticles and their subsequent removal to induce porosity $^{11}$ or by construction of a nano-structured architecture with controllable activated nanogels as nano-crosslinkers ${ }^{12}$. One of the most effective strategies to increase the deswelling rate of PNiPAAm gels has been the use of semi-interpenetrating networks (semi-IPNs) ${ }^{13,14}$ Semi-IPNs are composed of two or more chemically distinct networks wherein one of the polymer components is cross-linked while the other is in its linear form. For example, the reaction of crosslinking of PNiPAAm in the presence of alginate gives rise to semi-IPNs of alginate and PNiPAAm (Alg-PNiPAAm) that present an increased deswelling rate with respect to pure PNiPAAm as a result of a more homogeneous pore size distribution ${ }^{15}$.

Interestingly, the in situ synthesis of iron oxide nanoparticles by a process of coprecipitation of iron salts in alkaline media within Alg-PNiPAAm semi-IPNs gives rise to polymer hydrogels with response to temperature and magnetic fields ${ }^{16,17}$. The oxidation procedure can be repeated several times in order to increase the iron oxide content. The structural organization of iron oxide nanoparticles within the nanocomposite hydrogels has been determined by small angle $\mathrm{X}$ ray scattering (SAXS). For nanocomposite gels, the scattering contrast is given by the difference in electron density between the iron oxide nanoparticles and the polymer matrix. The pristine polymer matrix (with no nanoparticles) does not provide a scattering pattern. Results 
showed that the polymeric matrix acts as a template that controls the size and size distribution of iron oxide nanoparticles ${ }^{18}$. Further information can be retrieved from ultra-small angle X-ray scattering (USAXS) that provides information on the particle size distribution of iron oxide nanoparticles within hydrated nanocomposite gels. Fitting of USAXS scattering patterns to the so called unified model allows determining the size of particle aggregates which does not increase with the number of oxidation cycles ${ }^{19}$.

The freeze-drying of Alg-PNiPAAm semi-IPNs and its nanocomposites with iron oxide nanoparticles results in porous materials designated as aerogels. We have recently reported on the dynamics of nanocomposite aerogels as determined through X-rayphotocorrelation spectroscopy $(\mathrm{XPCS})^{20}$. Samples presented a porous structure as a consequence of the process of freeze-drying of the hydrated semi-IPNs. A hyperdiffusive dynamics at room temperature was found for all of them that was attributed to their characteristic porous structure and not to the presence of nanoparticles. This hyperdiffusive behavior resembles very much that encountered for jammed systems like colloidal suspensions $^{21-23}$. In this context, the complex porous structure characteristic of these aerogels can be contemplated as a jammed system ${ }^{7,}{ }^{24}$ whose dynamics, as revealed by XPCS, can be associated to internal fluctuations of the pore walls.

In this paper we investigate dynamics in hydrated Alg-PNiPAAm gels and their nanocomposites during the process of deswelling starting from temperatures below the LCST of the material to temperatures above the LCST of the material by means of XPCS measurements. Deswelling kinetic experiments of the hydrogels in water will also be conducted above the LCST and the corresponding kinetic curves will be determined. The effect of the presence of iron oxide nanoparticles on the deswelling kinetic dynamics of nanocomposite Alg-PNiPAAm gels will be discussed and reported here. 


\section{EXPERIMENTAL SECTION}

\section{Materials}

The N-Isopropylacrylamide (N-AAm) (Panreac), the initiator potassium persulfate (Fluka), the crosslinker $N, N^{\prime}$-Methylenebisacrylamide (Bis) (Aldrich), and the accelerator $N, N, N^{\prime}, N^{\prime}$-Tetramethylethylenediamine (TEMED) (Bio-Rad) were used as received. Alginic acid sodium salt from brown algae with a $65-70 \%$ guluronnic acid and $\mathrm{Mw}=100-200 \mathrm{kDa}$ according to the manufacturer was purchased from Aldrich and used as received. Alginate stock solutions were prepared by dissolving alginate powder in distilled water to yield $1 \mathrm{~g} / 100 \mathrm{~mL}$ solutions. Ferrous chloride $\left(\mathrm{FeCl}_{2} \cdot 4 \mathrm{H}_{2} \mathrm{O}\right)$, ferric chloride $\left(\mathrm{FeCl}_{3} \cdot 6 \mathrm{H}_{2} \mathrm{O}\right)$ were purchased from Aldrich and used as received.

\section{Preparation and characterization of gels}

Semi-interpenetrating (semi-IPN) polymer networks constituted by alginate and poly (N-isopropylacrylamide) (Alg-PNiPAAm) were obtained by polymerizing $3 \mathrm{~g}$ of $\mathrm{N}$ AAm and $0.15 \mathrm{~g}$ of Bis in $40 \mathrm{~mL}$ of an $1 \mathrm{wt}-\%$ alginate aqueous solution. The molar percentage of crosslinker with respect to monomer thus achieved was 3 wt.- $\%$. The polymerization was initiated by the potassium persulfate/TEMED redox system and carried out at room temperature. Solutions were poured out into Petri dishes and allowed to react at room temperature for $24 \mathrm{~h}$. Samples obtained were dialyzed against fresh water during 2 days to remove all the unreacted monomer and designated as AlgPNiPAAm (blank).

For the preparation of nanocomposite hydrogels, cylindrically shaped specimens of 20 $\mathrm{mm}$ in diameter were cut from the Alg-PNiPAAm (blank) samples and immersed in 200 $\mathrm{mL}$ aqueous solution containing $2.1 \mathrm{~g} \mathrm{FeCl}_{2} .4 \mathrm{H}_{2} \mathrm{O}$ and $5.8 \mathrm{~g} \mathrm{FeCl}_{3} \cdot 6 \mathrm{H}_{2} \mathrm{O}$ and maintained under constant $\mathrm{N}_{2}$ bubbling for 4 hours. After that period of time, yellowish gels were obtained that were immersed in a $0.5 \mathrm{M}$ ammonium hydroxide solution $\left(\mathrm{NH}_{3}\right.$ 
30 wt. \%) and maintained under constant $\mathrm{N}_{2}$ bubbling for 30 minutes. The resulting black gels were washed with distilled water for 24 hours. The procedure was repeated up to three times. Thermogravimetric measurements were performed on dried gels on a Q500 TA Instruments TGA, using a nitrogen stream as the purge gas, at a heating rate of $10{ }^{\circ} \mathrm{C} / \mathrm{min}$ over the range $40-900{ }^{\circ} \mathrm{C}$. Considering that the Alg-PNiPAAm (blank) decomposes completely at $900{ }^{\circ} \mathrm{C}$, the residual weight obtained for the samples with iron oxide nanoparticles (NPs) can be taken as an indication of the iron oxide content in the samples ${ }^{16}$. Nanocomposite hydrogel samples were designated as FeAlg-PNiPAAm (15) and FeAlg-PNiPAAm (22) where the numbers in brackets denote the iron oxide content, 15 wt. $\%$ and 22 wt. $\%$ respectively as determined through TGA measurements.

Diameters corresponding to primary particles and aggregates formed by primary particles were determined from model fits to ultra-small angle X-ray scattering as previously reported for samples prepared following the same procedure. The size of the individual spherical nanoparticles was $\sim 20 \mathrm{~nm}$ for the nanocomposite hydrogels and both samples presented the same degree of dispersion of the individual nanoparticles with an aggregate size of $\sim 100 \mathrm{~nm}^{19}$.

The swelling ratios of the gels were measured gravimetrically at $\mathrm{T}=20^{\circ} \mathrm{C}$. Samples were weighed after being incubated in deionized water for $24 \mathrm{~h}$ at $\mathrm{T}=20{ }^{\circ} \mathrm{C}\left(W_{s}\right)$. Afterwards, samples were freeze-dried to eliminate water and the dry weight of the gel $\left(W_{d}\right)$ was recorded. The swelling ratio defined as, Swelling ratio $=\frac{W_{s}}{W_{d}}$, is reported for the three samples under study in table 1. 
Table 1. Characteristics of the samples under study

\begin{tabular}{ccc}
\hline Sample name & $\begin{array}{c}\text { Iron oxide } \\
\text { content (Wt. \%) }\end{array}$ & Swelling ratio \\
\hline Alg-PNiPAAm (blank) & 0 & 20 \\
\hline FeAlg-PNiPAAm (15) & 15 & 14 \\
\hline FeAlg-PNiPAAm (22) & 22 & 11 \\
\hline
\end{tabular}

\section{Deswelling measurements}

The kinetics of deswelling of the hydrogels was measured gravimetrically at $37^{\circ} \mathrm{C}$. Gels were allowed to swell to equilibrium in distilled water at $20{ }^{\circ} \mathrm{C}$, and then the swollen gels were transferred into distilled water at $37{ }^{\circ} \mathrm{C}$. Sample surfaces were wiped with moistened filter paper to remove water and the weight during collapsing was recorded at different times $\left(W_{t}\right)$. Water retention $(\%)$ is defined as:

$$
\text { Water retention }(\%)=100 * \frac{W_{t}}{W_{s}}
$$

where $W_{s}$ corresponds to the weight of the fully swollen hydrogels.

\section{X-ray photocorrelation spectroscopy}

XPCS experiments were performed at the beam line P10 of PETRA III using $7 \mathrm{keV}$ xrays. A pixelated 2D detector (Princeton Instruments PI-LCX), located at $5.08 \mathrm{~m}$ downstream of the sample, was used to record the scattering patterns. The detector consists of $1340 \times 1300$ pixels each having an area of $20 \times 20 \mu \mathrm{m}^{2}$. A q range from 0.018 to $0.12 \mathrm{~nm}^{-1}$ was covered. For every measurement, 400 frames with an acquisition time of $0.1 \mathrm{~s}$ and a delay time (waiting time between two consecutive frames) of $0.9 \mathrm{~s}$ were recorded. In order to control temperature inside the test chamber, a cryostat was connected to the sample holder. Sealed glass capillars (Hilgenberg) were employed as sample containers for all the experiments. 
Experimentally, we measure the normalized intensity-intensity autocorrelation function $\mathrm{g}^{(2)}(\mathrm{q}, \mathrm{t})$, which is related to the intermediate scattering function (ISF, $f(\mathrm{q}, \mathrm{t})$ ) as:

$$
g^{(2)}(q, t)=1+b[f(q, t)]^{2}
$$

where $b$ and $t$ are the instrument dependent Siegert factor and the delay time respectively $^{25,26}$ 


\section{RESULTS AND DISCUSSION}

Alg-PNiPAAm semi-IPNs and their nanocomposites present a LCST below which the gels are fully swollen and above which they are collapsed. The LCST determined as the maximum in the endotherm observed in DSC experiments for Alg-PNiPAAm (blank) is $34^{\circ} \mathrm{C}$ and slightly decreases to $32^{\circ} \mathrm{C}$ and $31^{\circ} \mathrm{C}$ for the nanocomposite hydrogels, FeAlgPNiPAAm (15) and FeAlg-PNiPAAm (22) ${ }^{16}$. In order to fully investigate the dynamics of the samples under study as a function of temperature, XPCS experiments were performed on samples equilibrated at $\mathrm{T}=22^{\circ} \mathrm{C}(\mathrm{T}<\mathrm{LCST})$ and at $\mathrm{T}=37^{\circ} \mathrm{C}(\mathrm{T}>\mathrm{LCST})$. The results corresponding to XPCS experiments performed on samples Alg-PNiPAAm (blank), and FeAlg-PNiPAAm (22) measured at $\mathrm{T}=22{ }^{\circ} \mathrm{C}$ are shown in figure 1. Here, a series of simultaneously measured intensity autocorrelation functions for different values of transfer momentum $\mathrm{q}$ is shown. Both samples present a dynamic process at $\mathrm{T}=22{ }^{\circ} \mathrm{C}$ in the time scale investigated by XPCS. It is also clear that the dynamics becomes slower as the value of $q$ decreases.

Numerous studies ${ }^{25,27,28}$ have reported that the ISF, and consequently $g^{(2)}(q, t)$ are best described by means of a Kohlrausch-Williams-Watts (KWW) expression:

$$
g^{(2)}(q, t)=B+A \exp \left(-2\left[\frac{t}{\tau(q)}\right]^{\beta(q)}\right)
$$

where $\beta$ is the Kohlrausch exponent denoting the deviation from a single Debye relaxation $(\beta=1)$ and $\tau$ is the relaxation time of the process, $\mathrm{B}$ is the baseline and $\mathrm{A}$ is associated to the contrast. The data in figure 1 can be well described by eq. 3 assuming KWW exponents values $\beta>1$, which indicate a strong non-exponential character of the relaxation. 

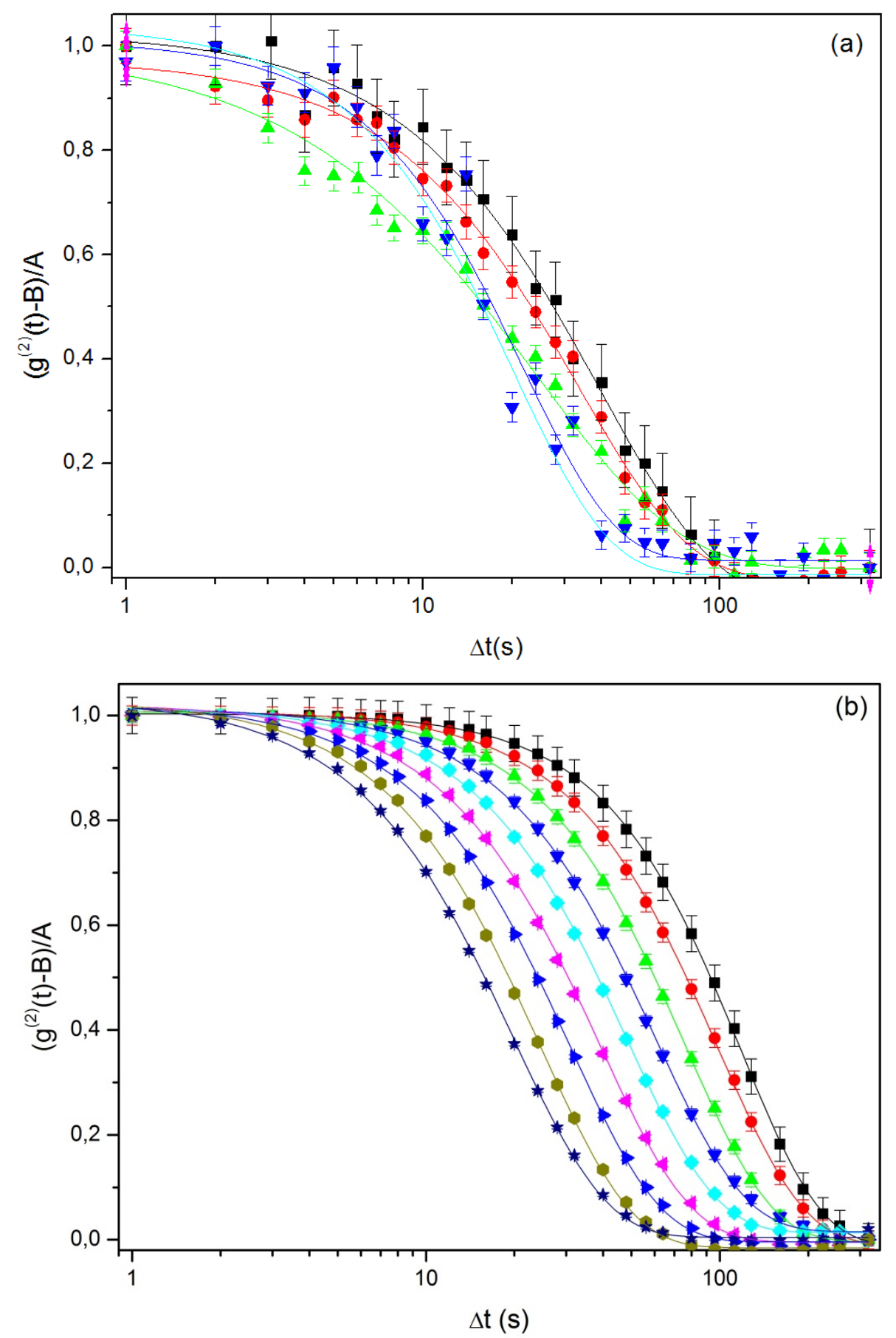

Figure 1. XPCS intensity correlation functions taken at room temperature for different values of transfer momentum q. (a) Alg-PNiPAAm (blank) and (b) FeAlgPNiPAAm (22). (घ) $\mathrm{q}=0.0205 \mathrm{~nm}^{-1},(\bullet) \mathrm{q}=0.0257 \mathrm{~nm}^{-1},(\Delta) \mathrm{q}=0.0321 \mathrm{~nm}^{-1},(\nabla)$ $\mathrm{q}=0.0402 \mathrm{~nm}^{-1}$, () $\mathrm{q}=0.0503 \mathrm{~nm}^{-1},(<) \mathrm{q}=0.0629 \mathrm{~nm}^{-1},(\boldsymbol{\Delta}) \mathrm{q}=0.0787 \mathrm{~nm}^{-1}$ and (hexagon) $\mathrm{q}=0.0981 \mathrm{~nm}^{-1}$ and (star) $\mathrm{q}=0.121 \mathrm{~nm}^{-1}$. Continuous lines are fits to equation 2. Note that for sample Alg-PNiPAAm (blank) only correlation functions taken at $\mathrm{q} \geq 0.0402 \mathrm{~nm}^{-1}$ are presented. 
Figure 2 a shows the $\mathrm{q}$-dependence of $\tau$ extracted from the fittings of $g^{(2)}(\mathrm{q}, \mathrm{t})$ (eq.3) to our data in double logarithmic scale for the three samples under study. Dotted lines in figure $2 \mathrm{a}$ indicate the fits to a power-law form of the type $\tau \sim \mathrm{q}^{-1}$ in the experimental data accessible in the q-range under study. This dependence is indicative of ballistic dynamics $^{29}$. In addition, the slowdown of the dynamics in the nanocomposite hydrogels with respect to the blank sample is confirmed at temperatures below LCST. It is interesting to note that this result was recently reported for freeze-dried aerogels for which the dynamics of the nanocomposite materials slowed down with respect to the sample with no nanoparticles ${ }^{20}$. We have previously demonstrated that iron oxide nanoparticles are bound to the polymer matrix thus influencing the swelling and mechanical properties of hydrated samples ${ }^{19}$. Therefore the increase of the relaxation times in the nanocomposite hydrogels reflects the entropic penalty that the polymer matrix suffers as a consequence of the establishment of interactions with the nanoparticles. On the other hand, the increase of nanoparticles content does not have an influence on the dynamics of the resulting nanocomposite gels.

A representation of the parameter $\beta$ as a function of $\mathrm{q}$ extracted from the fitting to equation 3 is shown in figure $2 b$. The $\beta$ values do not exhibit a clear dependence on $q$ but the average value seems to increase with the nanoparticle content. That is, AlgPNiPAAm (blank) present $\beta$ values close to 1.2 whereas these values increases to 1.5 and 1.7 for samples FeAlg-PNiPAAm (15) and sample FeAlg-PNiPAAm (22) respectively. It is clear that the slow relaxation behavior observed here for both the hydrogel and its nanocomposites present an hyper-diffusive behavior resembling very much that encountered for colloidal suspensions ${ }^{21,30,31}$. The fact that the sample AlgPNiPAAm (blank) presents a dynamic process of the same characteristics as the nanocomposite hydrogels suggests that the hyper-diffusive dynamics arises from the 
characteristic porous structure of the gel and hence it cannot be attributed to the nanoparticles per se. As previously reported, this hyper-diffusive dynamics was also found when these hydrogels were subjected to a process of freeze-drying thus preserving their porous microstructure ${ }^{20}$. The pore sizes obtained from analysis of SEM images corresponding to freeze dried aerogels are $\sim 5 \mu \mathrm{m}$. This morphology is maintained in the hydrated samples. It is important to note that, in the case of freezedried aerogels, the relaxation times obtained were one order of magnitude slower than the corresponding to hydrated gels shown in figure $2 \mathrm{a}^{20}$. This suggests that water has an effect in accelerating the dynamics of the hydrogels.

As previously reported ${ }^{6}$, hyper-diffusive dynamics is also characteristic of PNiPAAm gels at temperatures above LCST. The relaxation times $\tau$ and the compressed exponent $\beta$ is also plotted in figure 2 for sample FeAlg-PNiPAAm (22) measured at $\mathrm{T}=37{ }^{\circ} \mathrm{C}$ (T>LCST). The results show a power-law form of the type $\tau \sim q^{-1}$ which is indicative of hyper-diffusive dynamics and $\beta$ values $\sim 1.7$. The deswelling process is a kinetic process, that is, at a certain temperature above LCST, the gel volume decreases linearly with time until the equilibrium volume is reached ${ }^{6}$. The fact that XPCS experiments were carried out immediately after the sample FeAlg-PNiPAAm (22) reached $\mathrm{T}=37^{\circ} \mathrm{C}$, might explain why the relaxation times obtained for the sample at room temperature $(\mathrm{T}<\mathrm{LCST})$ and at $\mathrm{T}=37^{\circ} \mathrm{C}(\mathrm{T}>\mathrm{LCST})$ are within the experimental error. Further insight on the dynamics of the samples as a function of temperature is shown ahead. 


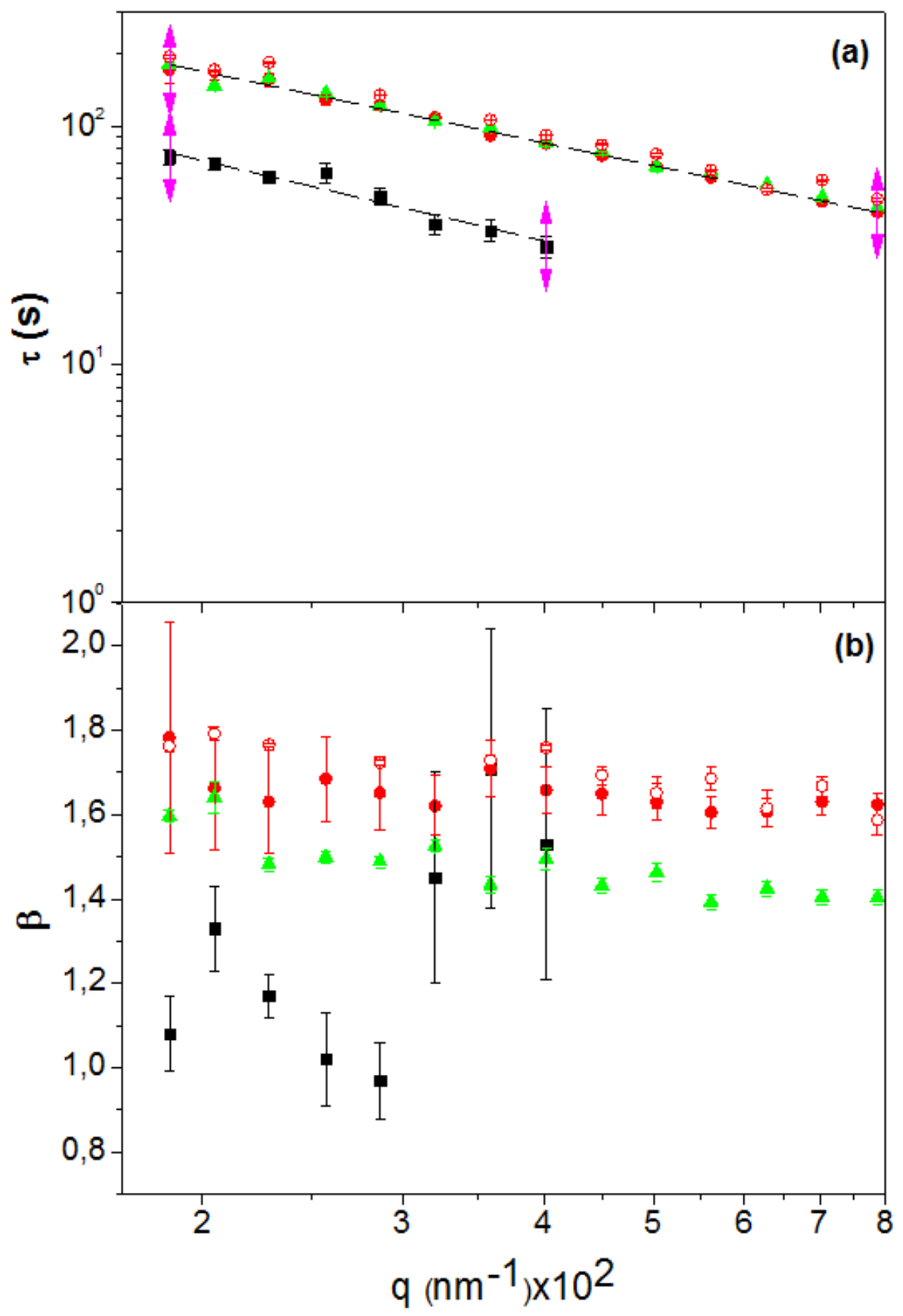

Figure 2. (a) Relaxation time plotted against q on a log-log scale (dashed lines are linear fits) and (b) compressed exponent $\beta$ vs. $q$ for the three samples under consideration: (匹) Alg-PNiPAAm (blank), ( $\triangle$ ) FeAlg-PNiPAAm (15) and (•) FeAlgPNiPAAm (22). Open symbols (O) correspond to data obtained for sample FeAlgPNiPAAm (22) measured at $\mathrm{T}=37^{\circ} \mathrm{C}$. 
The effect of the presence of NPs on the dynamics of Alg-PNiPAAm gels at temperatures above LCST was further investigated in samples equilibrated at $\mathrm{T}=37^{\circ} \mathrm{C}$. As a representative example, the intensity autocorrelation functions obtained at a fixed $\mathrm{q}$ $\left(q=0.0402 \mathrm{~nm}^{-1}\right)$ corresponding to the three samples under study are shown in figure 3 .

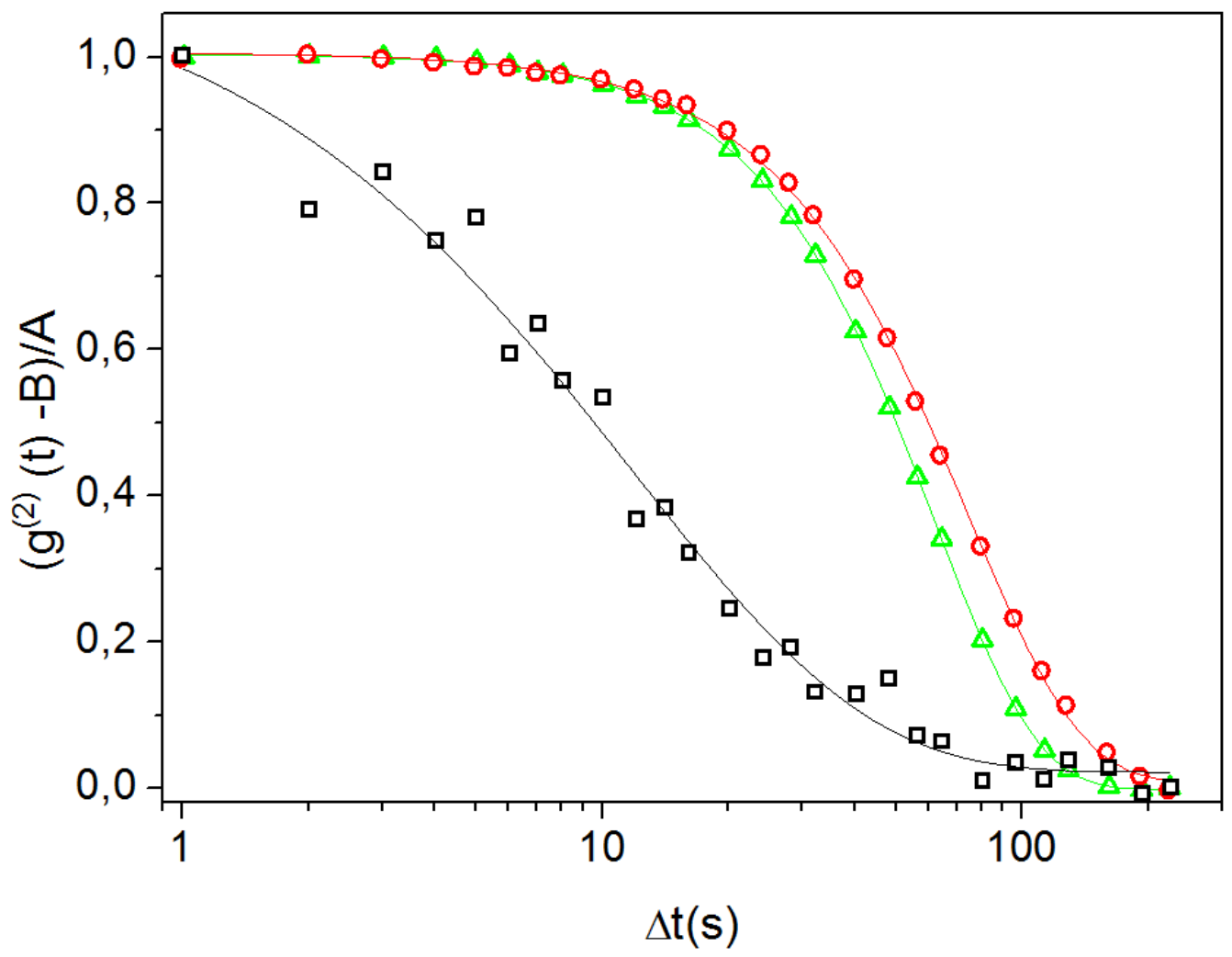

Figure 3. XPCS intensity correlation functions taken at $q=0.0402 \mathrm{~nm}^{-1}$ and $T=37{ }^{\circ} \mathrm{C}$ for ( $\square$ ) Alg-PNiPAAm (blank), ( $\triangle$ ) FeAlg-PNiPAAm (15) and (O) FeAlg-PNiPAAm (22)

Similar to the dynamics found for samples below LCST, the presence of iron oxide nanoparticles slows down the dynamics at temperatures above LCST. All the curves can be fitted to equation 3 with $\beta$ values higher than 1 which indicates an hyper-diffusive behaviour of the relaxation of the gels at $\mathrm{T}=37^{\circ} \mathrm{C}$. The relaxation times obtained are 34 s, $91 \mathrm{~s}$ and $115 \mathrm{~s}$ for Alg-PNiPAAm (blank), FeAlg-PNiPAAm (15) and FeAlgPNiPAAm (22) respectively. Assuming that the dynamics found by XPCS at temperatures above LCST originate from the intrinsic porous gel structure, the results found can be related to those obtained from measuring the deswelling rate of the 
hydrogels at temperatures higher than $\mathrm{LCST}^{6}$. For our samples, deswelling curves were carried out at $\mathrm{T}=37^{\circ} \mathrm{C}$ and results are represented in figure 4 .

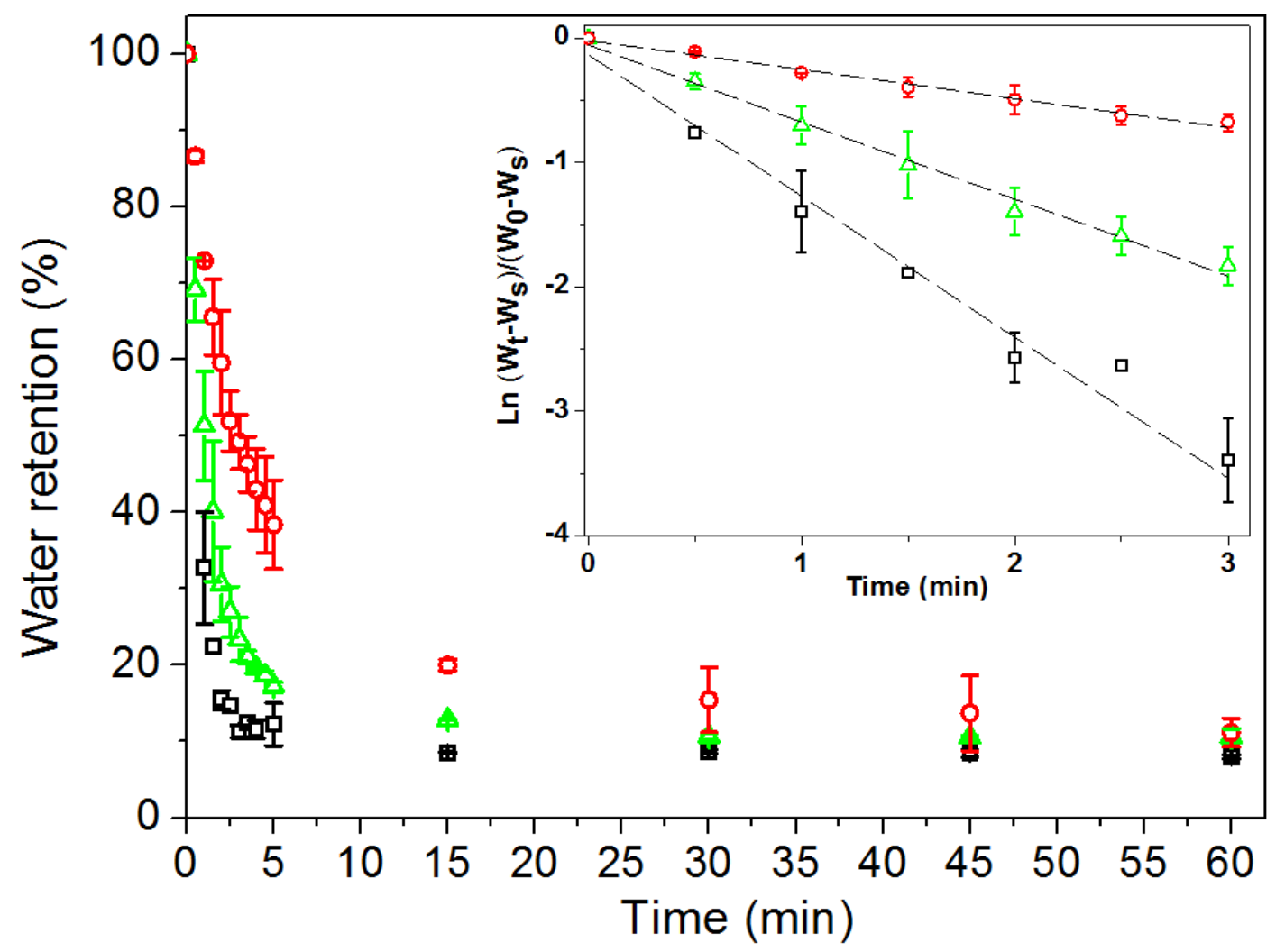

Figure 4. Deswelling kinetics of the hydrogels under study in deionized water at $37 \mathrm{C}$. The inset shows the rate analysis of the deswelling properties of the hydrogels. ( $\square$ ) AlgPNiPAAm (blank), ( $\triangle$ ) FeAlg-PNiPAAm (15) and (O) FeAlg-PNiPAAm (22)

Compared to sample Alg-PNiPAAm (blank), the nanocomposite gels FeAlg-PNiPAAm (15) and FeAlg-PNiPAAm (22) exhibited lower deswelling kinetics. Alg-PNiPAAm (blank) lost over $90 \%$ of its water content after 5 min whereas, during the same time, nanocomposite gels, FeAlgPNiPAAm (15) and FeAlg-PNiPAAm (22), loosed $~ 85 \%$ and $\sim 60 \%$ of their water content, respectively.

In order to analyze the deswelling process quantitatively, a semilogarithmic plot as a first-order rate analysis was applied to the time dependence of the deswelling as follows 11,32 : 


$$
\operatorname{Ln} \frac{\left(W_{t}-W_{s}\right)}{\left(W_{0}-W_{s}\right)}=-k t
$$

where $k$ is the deswelling rate constant and $t$ is the time. The greater the slope of the plot of $\operatorname{Ln}\left(W_{t}-W_{S}\right) /\left(W_{0}-W_{S}\right)$ against $t$, the faster the deswelling process.

The representation of the rate analysis of the deswelling properties of the three samples under study is shown in the inset of figure 4. As it can be observed, plots can be linearly fitted which indicates that the deswelling process is of first order. The obtained deswelling rate constants decrease from $0.018 \mathrm{~s}^{-1}$ for Alg-PNiPAAm (blank) to 0.0039 $\mathrm{s}^{-1}$ for FeAlg-PNiPAAm (22). This implies that the presence of iron oxide NPs in the nanocomposite gels slows down the deswelling kinetics. As previously reported, during the process of formation of iron oxide NPs inside Alg-PNiPAAm gels, some partial hydrolysis of alginate chains takes place leading to the collapse of the porous structure $^{16}$. This change in the porous structure of the gels together with the fact that the iron oxide nanoparticles are linked to the polymer chains ${ }^{19}$ might be responsible for the slowdown of the deswelling kinetics observed for the nanocomposite gels which, at the same time, determines longer relaxation times as determined through XPCS measurements.

In order to deepen on the dynamics of the samples as a function of temperature, XPCS experiments were carried out on the three samples under study equilibrated at temperatures ranging from $\mathrm{T}=22{ }^{\circ} \mathrm{C}$ to $\mathrm{T}=42{ }^{\circ} \mathrm{C}$. As a representative example, Figure 5 shows the intensity autocorrelation functions at a fixed $\mathrm{q}=0.0412 \mathrm{~nm}^{-1}$ for the nanocomposite hydrogel FeAlg-PNiPAAm (15) at different temperatures. As it can be observed, there is a dynamic process at all the temperatures under investigation in the 
time scale investigated by XPCS. Besides, there is a change in the temperature dependence of the dynamics as temperature increases.

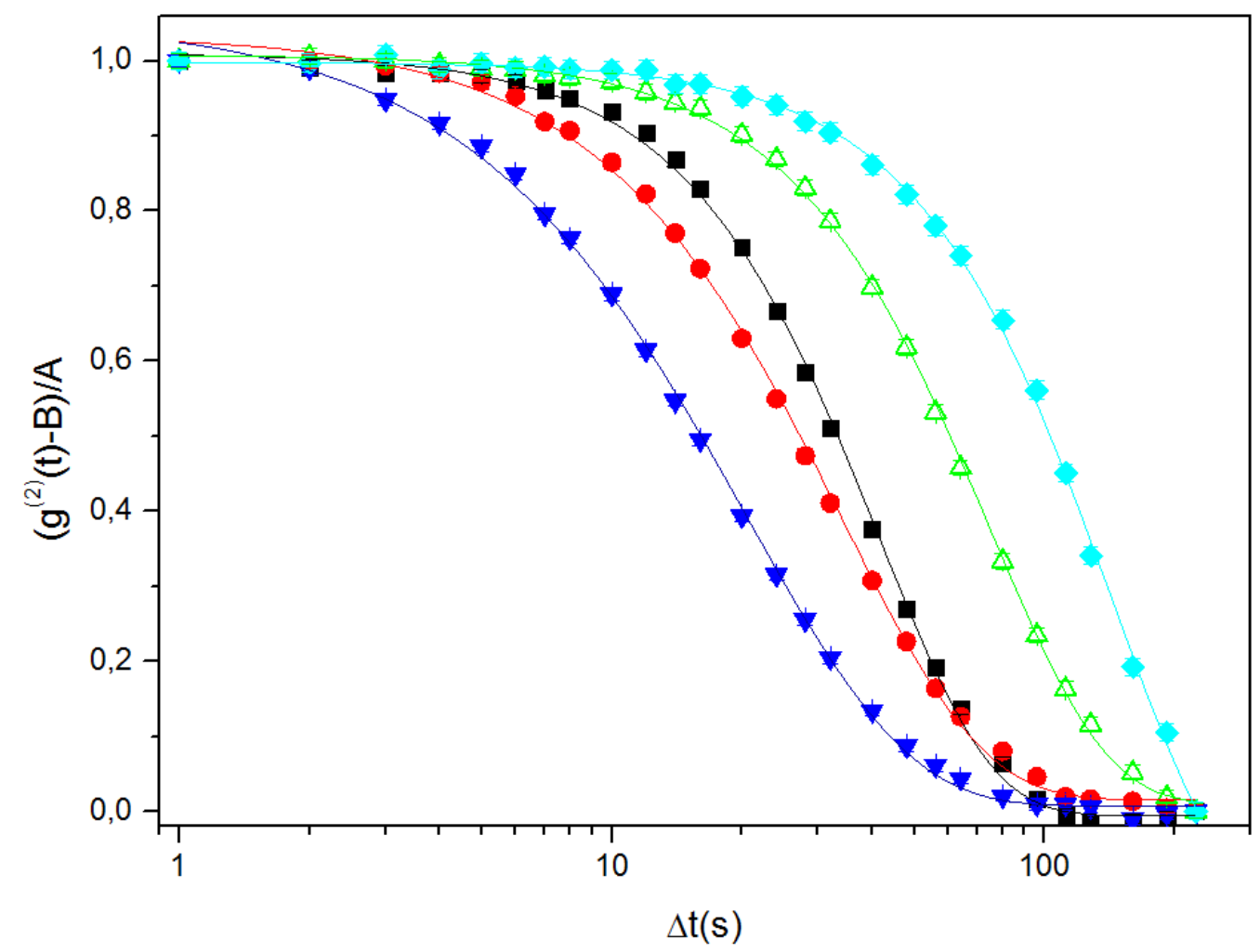

Figure 5. XPCS intensity correlation functions taken at $\mathrm{q}=0.0402 \mathrm{~nm}^{-1}$ for FeAlgPNiPAAm (15) and different temperatures (घ) T=22 ${ }^{\circ} \mathrm{C},(\bullet) \mathrm{T}=27^{\circ} \mathrm{C},(\boldsymbol{\nabla}) \mathrm{T}=32{ }^{\circ} \mathrm{C}$, $(\triangle) \mathrm{T}=37^{\circ} \mathrm{C}$ and $(\diamond) \mathrm{T}=42^{\circ} \mathrm{C}$

The relaxation times extracted from the fitting to equation 3 of the intensity autocorrelation functions depicted in figure 5 are represented as a function of temperature for the three samples under study in figure 6 . As it can be observed, the relaxation times remain almost constant at temperatures lower than $\mathrm{T}=32{ }^{\circ} \mathrm{C}$ and from that temperature, relaxation times start to increase. At $\mathrm{T}=32{ }^{\circ} \mathrm{C}$, there is a minimum in the relaxation time determined by XPCS which is consistent with the LCST of the materials as determined through DSC experiments ${ }^{16}$. 


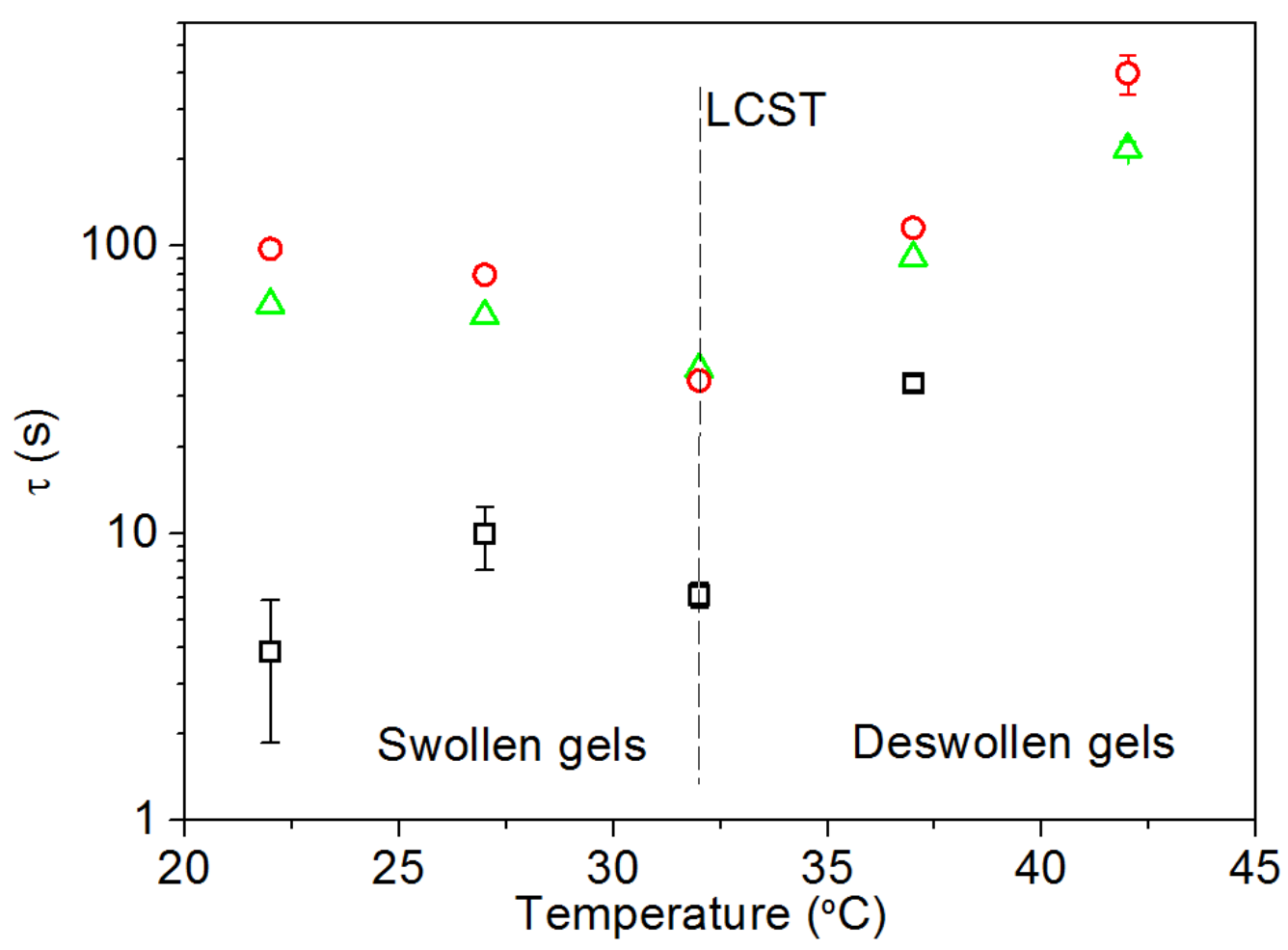

Figure 6. Relaxation times $(\tau)$ plotted as a function of temperature for $(\square)$ AlgPNiPAAm (blank), ( $\triangle$ ) FeAlg-PNiPAAm (15) and (O) FeAlg-PNiPAAm (22) measured at $\mathrm{q}=0.0402 \mathrm{~nm}^{-1}$.

Further information can be retrieved from the two-time correlation function $\mathrm{G}(\mathrm{q}, t 1, t 2)$ 33-35.

$$
G(q, t 1, t 2)=\frac{\langle I(q, t 1) I(q, t 2)\rangle_{p i x}}{\langle I(q, t 1)\rangle_{p i x}\langle I(q, t 2)\rangle_{p i x}}
$$

Here, the ensemble averages are performed over many pixels of the CCD detector, all having the same q value. The variables to describe the two-time correlation function are the average time or age, $t_{a}=(t 1+t 2) / 2$, measured by the distance along the $t 1=t 2$ diagonal in the perpendicular direction and the time difference $t=t 1-t 2$, which is the distance from the $t 1=t 2$ diagonal in the perpendicular direction. 
Figure 7 shows the two-time correlation functions corresponding to the sample FeAlgPNiPAAm (22).
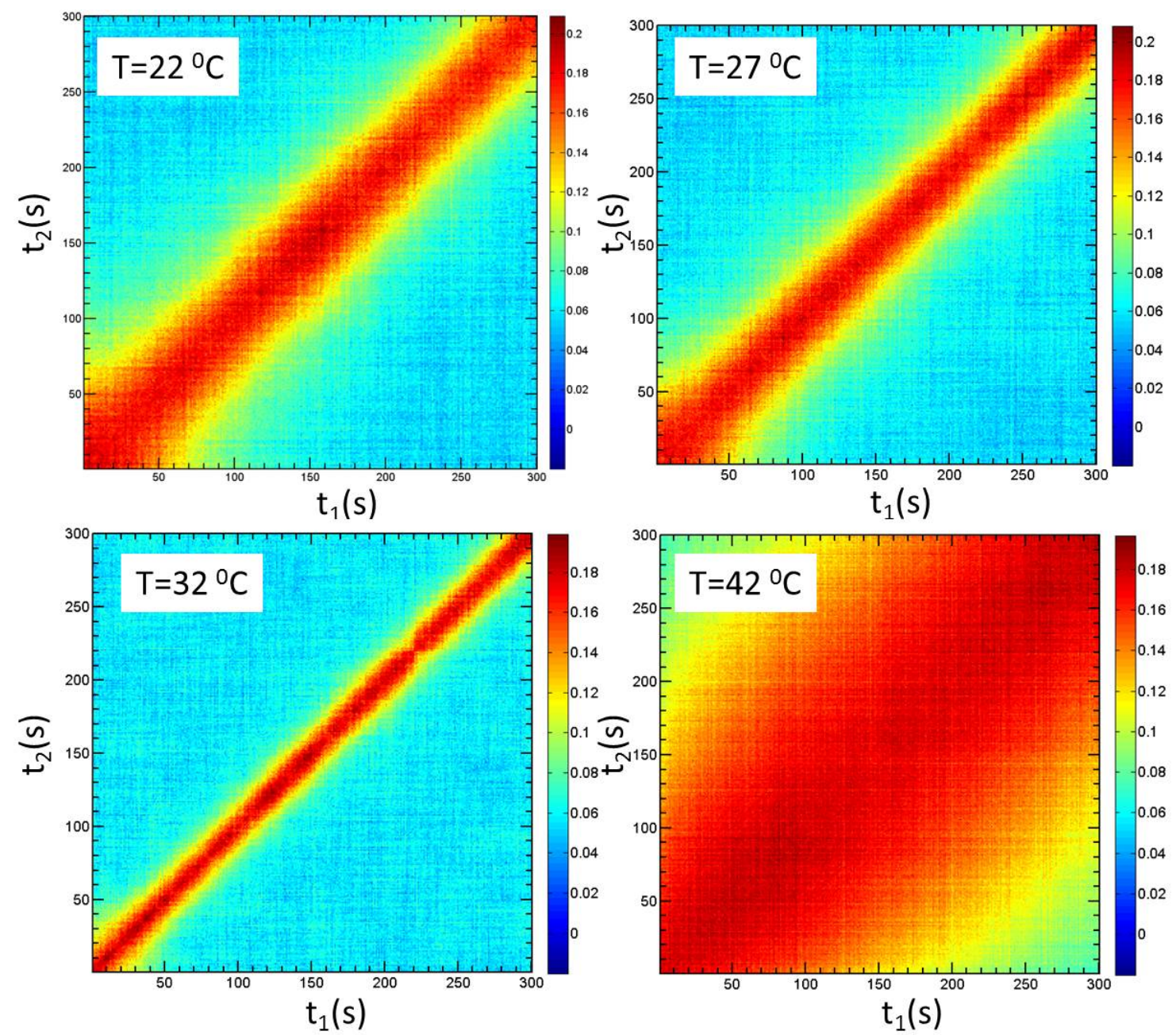

Figure 7. Two-time correlation function $\mathrm{G}\left(\mathrm{q}=0.0439 \mathrm{~nm}^{-1}, \mathrm{t} 1, \mathrm{t} 2\right)$ obtained for sample FeAlg-PNiPAAm (22) at different temperatures

At temperatures below LCST, the iso-intensity lines in plots $G(q, t 1, t 2)$ show a decrease in the width perpendicular to the diagonal which indicates an acceleration of the dynamics of the samples at these temperatures. At temperatures above LCST, there is an increase in the width perpendicular to the diagonal which indicates slowing-down of dynamics with time. At $\mathrm{T}=32^{\circ} \mathrm{C}$, the two time correlation function depend only on the time difference $t$, and hence the two time correlation contour lines are parallel which is indicative of stationary dynamics. 


\section{CONCLUSIONS}

In this paper XPCS was employed to directly quantify the slow dynamics of swollen gels derived from poly ( $\mathrm{N}$-isopropylacrylamide) and its nanocomposites with iron oxide nanoparticles. Similar to the results found for freeze-dried aerogels, a hyper-diffusive dynamics has been found for swollen gels that can be attributed to their characteristic porous structure. The dynamics of the swollen samples is faster than that of the freezedried ones suggesting that water has an effect in accelerating the dynamics of the hydrogels. We have proven that XPCS is a useful technique to characterize the deswelling process of temperature responsive polymer gels. The dynamics below LCST, when gels are fully swollen, is faster than above LCST, when gels are deswollen. This effect further emphasizes the effect of water on accelerating the dynamics of the polymer gels. Finally, we have demonstrated that the presence of iron oxide nanoparticles in nanocomposite gels tend to slow down the dynamics independently from temperature. At temperatures above LCST of the material, the dynamics found by XPCS can be related to a decrease of the deswelling rate for the nanocomposite gels.

\section{ACKNOWLEDGMENTS}

Synchrotron measurements were carried out in beamline P10 at the light source Petra III at DESY, Hamburg, Germany. We thank DESY for financial support. Additional financial support from MINECO (grants MAT2012-33517, MAT2011-23455 and MAT2011-24797) is gratefully acknowledged. Rebeca Hernández thanks MEC for a Ramon y Cajal contract and Miryam Criado acknowledges MEC for a FPU fellowship. 


\section{REFERENCES}

(1) Schild, H. G.Prog. Polym. Sci. 1992, 17, 163-249.

(2) Shibayama, M.; Morimoto, M.; Nomura, S.Macromolecules 1994, 27, 5060-5066.

(3) Schmaljohann, D.Adv. Drug Deliv. Rev. 2006, 58, 1655-1670.

(4) Hoffman, A. S.Adv. Drug Deliv. Rev. 2013, 65, 10-16.

(5) László, K.; Fluerasu, A.; Moussaïd, A.; Geissler, E.Macromol. Symp. 2011, 306-307, 27-

(6) Laszlo, K.; Fluerasu, A.; Moussaid, A.; Geissler, E.Soft Matter 2010, 6, 4335-4338.

(7) Jaeger, H. M.Soft Matter 2015, 11, 12-27.

(8) Zhang, X.; Zhuo, R.; Yang, Y.Biomaterials 2002, 23, 1313-1318.

(9) Kato, N.; Sakai, Y.; Shibata, S.Macromolecules 2003, 36, 961-963.

(10) Zhang, J. T.; Huang, S. W.; Xue, Y. N.; Zhuo, R. X.Macromol. Rap. Comm. 2005, 26, 1346-1350.

(11) Serizawa, T.; Wakita, K.; Akashi, M.Macromolecules 2001, 35, 10-12.

(12) Xia, L.-W.; Xie, R.; Ju, X.-J.; Wang, W.; Chen, Q.; Chu, L.-Y.Nat Commun 2013, 4.

(13) Zhang, X.-Z.; Chu, C.-C.J. Appl. Polym. Sci. 2003, 89, 1935-1942.

(14) Guilherme, M. R.; Campese, G. M.; Radovanovic, E.; Rubira, A. F.; Tambourgi, E. B.; Muniz, E. C.J. Memb. Sci. 2006, 275, 187-194.

(15) Zhang, G.-Q.; Zha, L.-S.; Zhou, M.-H.; Ma, J.-H.; Liang, B.-R.Colloid Polym Sci 2005, 283, 431-438.

(16) Hernández, R.; Mijangos, C.Macromol. Rap. Comm. 2009, 30, 176-181.

(17) Hernández, R.; Sacristán, J.; Asín, L.; Torres, T. E.; Ibarra, M. R.; Goya, G. F.; Mijangos, C.J. Phys. Chem. A. 2010, 114, 12002-12007.

(18) Hernández, R.; Sacristán, J.; Nogales, A.; Ezquerra, T. A.; Mijangos, C.Langmuir 2009, 25, 13212-13218.

(19) Hernandez, R.; Sacristan, J.; Nogales, A.; Fernandez, M.; Ezquerra, T. A.; Mijangos, C.Soft Matter 2010, 6, 3910-3917.

(20) Hernández, R.; Nogales, A.; Sprung, M.; Mijangos, C.; Ezquerra, T. A.J. Chem. Phys. 2014, 140, -.

(21) Cipelletti, L.; Manley, S.; Ball, R. C.; Weitz, D. A.Phys. Rev. Lett. 2000, 84, 2275-2278.

(22) Bandyopadhyay, R.; Liang, D.; Yardimci, H.; Sessoms, D.; Borthwick, M.; Mochrie, S.; Harden, J.; Leheny, R.Phys. Rev. Lett. 2004, 93, 228302.

(23) Kwasniewski, P.; Fluerasu, A.; Madsen, A.Soft Matter 2014, 10, 8698-8704.

(24) Caps, H.; Delon, G.; Vandewalle, N.; Guillermic, R. M.; Pitois, O.; Biance, A. L.; Saulnier, L.; Yazhgur, P.; Rio, E.; Salonen, A.; Langevin, D.Europhysics News 2014, 45, 22-25.

(25) Kim, D.; Srivastava, S.; Narayanan, S.; Archer, L. A.Soft Matter 2012, 8, 10813-10818.

(26) Leheny, R. L.Curr. Opin. Colloid Interface Sci. 2012, 17, 3-12.

(27) Orsi, D.; Cristofolini, L.; Baldi, G.; Madsen, A.Phys. Rev. Lett. 2012, 108, 105701.

(28) Guo, H.; Bourret, G.; Corbierre, M. K.; Rucareanu, S.; Lennox, R. B.; Laaziri, K.; Piche, L.; Sutton, M.; Harden, J. L.; Leheny, R. L.Phys. Rev. Lett. 2009, 102, 075702.

(29) Cipelletti, L.; Ramos, L.; Manley, S.; Pitard, E.; Weitz, D. A.; Pashkovski, E. E.; Johansson, M.Faraday Disc. 2003, 123, 237-251.

(30) Caronna, C.; Chushkin, Y.; Madsen, A.; Cupane, A.Phys. Rev. Lett. 2008, 100, 055702.

(31) Duri, A.; Autenrieth, T.; Stadler, L. M.; Leupold, O.; Chushkin, Y.; Grubel, G.; Gutt, C.Phys. Rev. Lett. 2009, 102, 145701.

(32) Zhao, Z. X.; Li, Z.; Xia, Q. B.; Bajalis, E.; Xi, H. X.; Lin, Y. S.Chem. Eng. J. 2008, 142, 263270.

(33) Madsen, A.; Leheny, R. L.; Guo, H.; Sprung, M.; Czakkel, O.New. J. Phys. 2010, 12, 055001.

(34) Fluerasu, A.; Moussaïd, A.; Madsen, A.; Schofield, A.Phys. Rev. E 2007, 76, 010401.

(35) Sutton, M.; Laaziri, K.; Livet, F.; Bley, F.Opt. Express 2003, 11, 2268-2277. 


\section{For Table of Contents use only.}

Deswelling of poly (N-isopropylacrylamide) hydrogels and their nanocomposties with iron oxide nanoparticles as revealed by X-ray photocorrelation spectroscopy (XPCS)

Rebeca Hernández, Miryam Criado, Aurora Nogales, Michael Sprung, Carmen Mijangos, Tiberio A. Ezquerra.

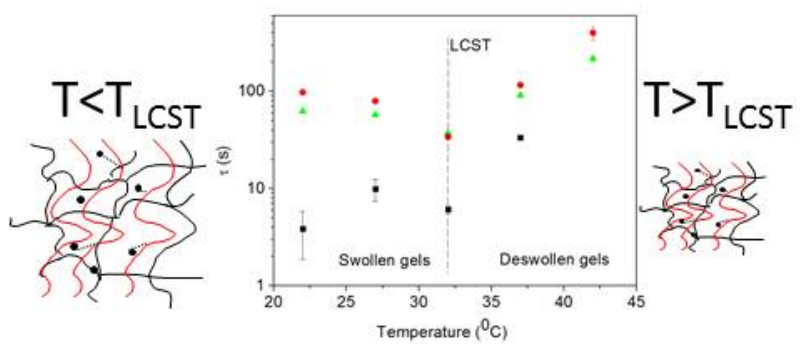

